\title{
Seismic Retrofit for Electric Power Systems
}

\section{Natalia Romero ${ }^{\text {a) }}$, Linda K. Nozick ${ }^{\text {a) }}$ Ian Dobson $^{\text {) }}$, Ningxiong $\mathrm{Xu}^{\text {a) }}$, Dean A. Jones ${ }^{\text {c) }}$}

This paper develops a two-stage stochastic program and solution procedure to optimize the selection of seismic retrofit strategies to increase the resilience of electric power systems to earthquake hazards. The model explicitly considers the range of earthquake events that are possible and, for each, an approximation to the distribution of damage that is experienced. This is important because electric power systems are spatially distributed; hence their performance is driven by the distribution of damage of the components. We test this solution procedure against the nonlinear integer solver in LINGO 13 and apply the formulation and solution strategy to the Eastern Interconnection where the seismic hazard stems from the New Madrid Seismic Zone.

\section{INTRODUCTION}

Earthquakes pose a significant risk to electric power systems as demonstrated by the following examples. On January 17, 1994 the Northridge earthquake struck the city of Los Angeles and surrounding areas. Two and a half million customers lost power (Dong et al. 2004). The Great Hanshin earthquake occurred a year later affecting the city of Kobe, Japan. Twenty fossil-fire power generation units, six $275 \mathrm{kV}$ substations, and two $154 \mathrm{kV}$ substations were damaged. Approximately, 2.6 million customers were affected by outages (Noda 2001). On May 18, 2008, the Wenchuan earthquake caused extensive damage to the local power transmission and distributions systems in the Sinchuan province, China. Approximately 900 substations and 270 transmission lines of the State Power Grid were damaged. It has been estimated that at least $90 \%$ of the damage could have been avoided by adopting new guidelines for seismic design (Eidinger 2009). 90\% of Chileans did not have electricity immediately following the February 27, 2010 8.8 MW earthquake. The event caused the largest power transmission company in Chile to have direct losses of

\footnotetext{
a) School of Civil and Environmental Engineering, Cornell University, Hollister Hall, Ithaca, NY 14850, USA

b) Department of Electrical and Computer Engineering, Iowa State University, Ames IA 50011, USA

c) Sandia National Laboratories, PO Box 5800, Albuquerque, NM 87185-1138, USA

Romero - 1
} 
approximately US \$ 6.5 billion (Long 2010). The devastating Tohoku Chiho - Taiheiyo-Oki earthquake on March 11, 2011 damaged 14 power plants, 70 transformers, and 42 transmission towers, among other failures. Outages stemming from the event affected 4.6 million residences and the April 7 aftershock affected an additional 4 million (Shumuta 2011).

This paper formulates an optimization model to select hardening-based mitigation measures for earthquake events and proposes a solution procedure for that optimization. The method is applied to address seismic risk in the Central United States. This region has received less attention than zones in the west but it has been the source of strong earthquakes in the past. In the winter of 1811-1812, a 7.8 MW intraplate earthquake centered in the New Madrid Seismic Zone (NMSZ) struck the central United States inducing liquefaction and permanent ground motion (Tuttle et al. 2002). It is important to notice that historic records of large earthquakes in the NMSZ suggest that the time between large events is somewhere between 200 to 800 years with an average of about 500 years. Considering that the last recorded high magnitude earthquake was in 1811-1812, we might be close to an event based on the low estimate of the recurrence relationship (Tuttle et al. 2002). In addition, due to soil conditions, ground shaking in this area is expected to affect a greater area than would be expected in California for a similar magnitude earthquake (Gomberg and Schweig 2007). Another characteristic to consider about the NMSZ is that since earthquakes are not as frequent as those on other faults, like the San Andreas, there may be an insufficient understanding of the earthquake risk in the area and, therefore, mitigation strategies in use may not be adequate.

The objective of this paper is to develop a model to optimize the selection of mitigation strategies in the electric power system to control the consequences of earthquake events. Research on mitigation against earthquake hazard has considered anchoring and reinforcement of electric power. Vanzi found optimized structural upgrading strategies for electric power networks using a new index to choose among critical nodes in the network (2000). The method was tested using a representation of the Sicily, Italy power network, which includes 181 nodes and 220 lines. Shumuta focused on upgrading substation equipment (2007). He evaluated the criticality of components with 4 indexes; two indexes represent earthquake resistance, the third index focuses on seismic performance, and the fourth index is cost-based. This method was tested on a hypothetical electric power system 
with 16 substations, located in the Nagoya region, Japan.

We implement a knapsack-based heuristic to solve the non-linear integer programming problem (NLIP) to optimize the selection of reinforcement strategies for electric power system components. For each mitigation strategy for each component we use an integer variable with an associated cost and estimate of risk reduction, if implemented. The selection of the investments using this structure under a budget limit yields a knapsack problem. A knapsack problem is a binary integer programming problem where the goal is to select a subset of items, each with a known cost, that yields the largest combined benefit and for which the combined cost of all items selected does not exceed the specified budget.

To model the seismic risk, we use a suite of earthquake scenarios that nearly replicates the estimated exceedance curves for peak ground acceleration (PGA) as given in the U.S. Geological Survey (USGS) Seismic Hazard Maps (USGS 2008b) at 81 control locations across the NMSZ. Since the electric power system is a spatially distributed system, we create a suite of consequence scenarios for each earthquake scenario, where each consequence scenario identifies the resulting damage state of each component. Once the damage state of a component is known, the expected time required for the component to be operational again and the cost of the repair can be estimated. The construction of these consequence scenarios provides an implicit representation of the distribution of damage for each earthquake scenario. The damage to the power grid considered is limited to transmission lines and substations. The operation of the power grid is modeled using an economic dispatch model. An economic dispatch model is an optimization model used to match electric power supply with demand in the lowest cost manner possible. We assume that the operator of the network has a limited budget to invest in mitigating the risk. The method is illustrated using the U. S. Eastern Interconnection power grid (EI); a network with more than 20,000 transmission lines and about 15,000 buses. A much simpler dataset for the NLIP is solved using LINGO 13 (Lindo System Inc. 2011). The results using LINGO and our proposed heuristic are compared for different mitigation budgets to gain a sense of the performance of the heuristic.

The next section develops the formulation. The third section presents the solution procedure (which is a heuristic). The fourth section describes the key elements of the case study. The fifth section describes the results of the application of the tools developed in sections two and three to the case study described in section four. It also includes a comparison of the performance of the solution procedure developed in the third section to

$$
\text { Romero - } 3
$$


LINGO 13 (Lindo System Inc. 2011), a commercial solver, for a very simplified problem instance. The sixth section summarizes the key elements of the paper and next steps for future research.

\section{FORMULATION}

We formulate this optimization problem as a two-stage stochastic program. A two-stage stochastic program is an optimization model formulation that incorporates uncertainty in the parameters of the model. The two-stage structure assumes that all decisions are made at one time instance prior to the resolution of all uncertainty. In this case, the uncertainty revolves around what damage will occur to each component in the electric power system. This uncertainty is expressed through the use of a number of consequence scenarios, where each consequence scenario gives the damage to each component. The decisions are made in what is termed the "first-stage" of the model. In our formulation, the first-stage is the identification of what components in the electric power system should be hardened. The consequences of those decisions, under each consequence scenario, occur in the "second-stage" of the model. In this problem formulation, the second-stage is the power flow across each component including what demands for power are not satisfied under each consequence scenario.

We first introduce the topology of the power network. Let $\Pi$ be the set of transmission lines. Let $S$ be the set of substations. Let $G$ be the set of generators. Let $B$ be the set of buses. Let $I(i)$ be the set of the electric power generators connected to bus $i$. We define the firststage binary decision variables as follows. Let $x_{s}^{D}=1$ if substation $s$ is reinforced and $x_{s}^{D}=0$ otherwise. The cost to reinforce substation $s$ is $b_{s}^{D}$. Let $y_{i j}^{D}=1$ if transmission line $(i, j)$ is reinforced and $y_{i j}^{D}=0$ otherwise. The cost to reinforce transmission line $(i, j)$ is $f_{i j}^{D}$. We assume that the total available budget is $M^{D}$. Since the total investment to reinforce the components cannot exceed the available budget, then equation (1) must hold.

$$
\sum_{s \in S} b_{s}^{D} x_{s}^{D}+\sum_{(i, j) \in \Pi} f_{i j}^{D} y_{i j}^{D} \leq M^{D}
$$

In this application, the cost of the seismic reinforcement of transmission lines is assumed to be a percentage of the total replacement cost of the line obtained from Balducci (Balducci et al. 2006). Seismic reinforcement of substations is defined as the anchorage of the transformers in the substations. Therefore the cost to reinforce a substation is estimated by 
multiplying the cost of anchoring a transformer (Shinozuka et al. 2003) by the number of transformers in the substation.

Based on the HAZUS seismic risk assessment methodology (FEMA 2003), five damage states are defined for electric power components: none, minor, moderate, extensive and complete. We choose to focus on the damage states none, moderate, extensive and complete because the damage associated with minor for substations is not substantial in this context. Moderate damage to substations generates a repair cost of about $40 \%$ of substation cost and does not affect any of the transformers in the substation. Extensive damage is assumed to imply damages costing $70 \%$ of the value of the substation including impacting $50 \%$ of the transformers in the substation. Complete damage, causes the complete loss of the substation including all the transformers. The estimated time for repair is 3 days for the moderate damage and a week for extensive damage. For complete damage, repairs can vary depending on the ease with which the transformers can be replaced. High voltage and/or customized transformers can have very large lead-times. Thus, for modeling purposes, we assume that for low voltage transformers, the operator would have access to spares within a month. We assume that the average lead-time for medium and high voltage transformers is 6 months. Therefore, all the components in substations under complete damage are back to normal within a month with the exception of medium and high voltage transformers, for which is 6 months. For transmission lines we only model two levels of damage: extensive and complete. Extensive damage for a transmission line corresponds to a damage ratio of $50 \%$ of the total cost of the line and complete damage results in costs totaling the full cost of the line. Transmission lines under extensive damage can be repaired within 3 days and under complete damage within a week. This implies that by the end of 6 months, in the worst case, the system is back to normal.

The repair times given above implies that we must divide the repair process into 4 time periods. The first period extends from the event to the end of the third day. By then, transmission lines that have experienced extensive damage have been restored. Also, substations under moderate damage have been repaired. The second time period extends from the beginning of day four to the end of the first week. By then, transmission lines that have experienced complete damage have been repaired, as well as substations under extensive damage. The third time period extends from the end of the first week to the end of the first month. By the end of this time period, low voltage transformers will have been

\section{Romero - 5}


replaced. The final time period extends from one month to six months. Six months after the event, medium and large voltage transformers will have been repaired. We define the following notation to encapsulate these time period definitions. Let $t_{0}=0, t_{1}=3$ days, $t_{2}=1$ week, $t_{3}=1$ month, and $t_{4}=6$ months, Then $t_{k}-t_{k-1}$ is the time length of period $k$ for $k=1,2,3,4$.

We assume that there are $N$ earthquake consequence scenarios, i.e., $n=1, \ldots, N$. The associated annual probability of scenario $n$ is $\operatorname{Pr}(n)$. Let $c^{B}$ be the cost per unit of demand which cannot be satisfied (per unit load shed cost). Let $c_{g}^{G}$ be the unit power generation cost of generator $g$. Note that the first-stage reinforcement decisions and the earthquake scenario determine the level of damage to each component; hence, the length of time from the earthquake that the component is unavailable and the repair cost is known. Let $\Psi_{s}^{n}\left(x_{s}^{D}\right)$ be the repair cost for substation $s$ under the first-stage decision $x_{s}^{D}$ for earthquake scenario $n$. Let $\Omega_{i j}^{n}\left(y_{i j}^{D}\right)$ be the repair cost of damaged transmission line $(i, j)$ under the first-stage decision $y_{i j}^{D}$ and given earthquake scenario $n$. Let $\Delta_{s}^{n k}\left(x_{s}^{D}\right)=1$ if substation $s$ is not functional in period $k$ under earthquake scenario $n$ for the given first-stage decision $x_{s}^{D}$ and $\Delta_{s}^{n k}\left(x_{s}^{D}\right)=0$ otherwise. Similarly, let $\Lambda_{i j}^{n k}\left(y_{i j}^{D}\right)=1$ if transmission line $(i, j)$ is not functional in period $k$ under scenario $n$ for the given first-stage decision $y_{i j}^{D}$ and $\Lambda_{i j}^{n k}\left(y_{i j}^{D}\right)=0$ otherwise. Notice that $\Lambda_{i j}^{n k}\left(y_{i j}^{D}\right)$ must be zero in periods 3 or 4 . Note that in this application, functions $\Psi_{s}^{n}\left(x_{s}^{D}\right)$ and $\Delta_{s}^{n k}\left(x_{s}^{D}\right)$ are nonlinear functions in $x_{s}^{D}$ and functions $\Omega_{i j}^{n}\left(y_{i j}^{D}\right)$ and $\Lambda_{i j}^{n k}\left(y_{i j}^{D}\right)$ are nonlinear functions in $y_{i j}^{D}$.

Now we define the second-stage decision variables. Let $\theta_{i}^{n k}$ be the phase angle in bus $i$ and in period $k$ under scenario $n$. Let $P_{i j}^{n k}$ be the real power flow in transmission line $(i, j)$ in period $k$ under scenario $n$. Since the electric flows can go in both directions, then $P_{i j}^{n k}$ can be either positive or negative. Let $G_{g}^{n k}$ be the nonnegative generation output from generator $g$ in period $k$ under scenario $n$. Let $U_{i}^{n k}$ be the nonnegative load shed in bus $i$ in period $k$ under scenario $n$. 
Let $m_{i j}$ be the reactance in transmission line $(i, j)$ and let $T_{i j}$ be an indicator with value 1 when the operator has a spare transformer for transmission line $(i, j)$ and 0 otherwise.

$$
\begin{aligned}
\left(\theta_{i}^{n 1}-\theta_{j}^{n 1}\right)\left(1-\Lambda_{i j}^{n 1}\left(y_{i j}^{D}\right)\right)\left(1-\Delta_{s_{i}}^{n 1}\left(x_{s}^{D}\right)\right)\left(1-\Delta_{s_{j}}^{n 1}\left(x_{s}^{D}\right)\right) & =m_{i j} P_{i j}^{n 1}, \forall(i, j), n \\
\left(\theta_{i}^{n 2}-\theta_{j}^{n 2}\right)\left(1-\Lambda_{i j}^{n 2}\left(y_{i j}^{D}\right)\right)\left(1-\Delta_{s_{i}}^{n 2}\left(x_{s}^{D}\right)\right)\left(1-\Delta_{s_{j}}^{n 2}\left(x_{s}^{D}\right)\right) & =m_{i j} P_{i j}^{n 2}, \forall(i, j), n \\
\left(\theta_{i}^{n 3}-\theta_{j}^{n 3}\right)\left(1-\Delta_{s_{i}}^{n 3}\left(x_{s}^{D}\right)\right)\left(1-\Delta_{s_{j}}^{n 3}\left(x_{s}^{D}\right)\right) & =m_{i j} P_{i j}^{n 3}, \forall(i, j), n \\
\left(\theta_{i}^{n 4}-\theta_{j}^{n 4}\right)\left(1-\Delta_{s_{i}}^{n 4}\left(x_{s}^{D}\right)\left(1-T_{i j}\right)\right) & =m_{i j} P_{i j}^{n 4}, \forall(i, j), n
\end{aligned}
$$

Constraints (2), (3), (4), and (5) approximate the active power flows on the transmission lines in the four periods of the repair process. If the per day demand at bus $i$ is $D_{i}$, then constraints (6) states flow conservation at each bus under each earthquake scenario.

$$
\sum_{g \in I(i)} G_{g}^{n k}-\sum_{(i, j) \in \delta^{+}(i)} P_{i j}^{n k}+\sum_{(i, j) \in \delta^{-}(i)} P_{i j}^{n k}=D_{i}-U_{i j}^{n k}, \forall i, k, n
$$

where $\delta^{+}(i)$ is the set of the transmission lines such that $(i, j) \in \Pi$ and $\delta^{-}(i)$ is the set of transmission lines such that $(j, i) \in \Pi$. Since the load shed at a bus cannot exceed the demand at the bus.

$$
0 \leq U_{i}^{n k} \leq D_{i}, \forall i, k, n
$$

We assume that generator $g$ has capacity $G_{g}^{m}$ and transmission line $(i, j)$ has capacity $P_{i j}^{m}$. Equations (8)-(12) reflect the capacity constraints in each generator and each transmission line in each time period under each earthquake scenario. Notice that the flow in a line goes to zero when it is connected to a non-operational component for a given scenario and time period

$$
\begin{gathered}
0 \leq G_{g}^{n k} \leq G_{g}^{m}, \forall g, k, n \\
\left|P_{i j}^{n 1}\right| \leq P_{i j}^{m}\left(1-\Lambda_{i j}^{n 1}\left(y_{i j}^{D}\right)\right)\left(1-\Delta_{s_{i}}^{n 1}\left(x_{s_{i}}^{D}\right)\right)\left(1-\Delta_{s_{j}}^{n 1}\left(x_{s_{j}}^{D}\right)\right), \forall(i, j), n \\
\left|P_{i j}^{n 2}\right| \leq P_{i j}^{m}\left(1-\Lambda_{i j}^{n 2}\left(y_{i j}^{D}\right)\right)\left(1-\Delta_{s_{i}}^{n 2}\left(x_{s_{i}}^{D}\right)\right)\left(1-\Delta_{s_{j}}^{n 2}\left(x_{s_{j}}^{D}\right)\right), \forall(i, j), n \\
\left|P_{i j}^{n 3}\right| \leq P_{i j}^{m}\left(1-\Delta_{s_{i}}^{n 3}\left(x_{s_{i}}^{D}\right)\right)\left(1-\Delta_{s_{j}}^{n 3}\left(x_{s_{j}}^{D}\right)\right), \forall(i, j), n
\end{gathered}
$$




$$
\left|P_{i j}^{n 4}\right| \leq P_{i j}^{m}\left(1-\Delta_{s_{i}}^{n 4}\left(x_{s_{i}}^{D}\right)\left(1-T_{i j}\right)\right), \forall(i, j), n
$$

where $s_{i}$ is the substation, to which bus $i$ belongs. The objective function of the two-stage stochastic program is to minimize the expected generation, load shed and repair costs in the four recovery periods as given in Equation (13).

$$
\sum_{n=1}^{N} \operatorname{Pr}(n) \sum_{k=1,2,3,4}\left(t_{k}-t_{k-1}\right)\left(\sum_{i \in B} c^{B} U_{i}^{n k}+\sum_{g \in G} c_{g}^{G} G_{g}^{n k}\right)+\sum_{s \in S} \Psi_{s}\left(x_{s}^{D}\right)+\sum_{(i, j) \in \Pi} \Omega_{i j}\left(y_{i j}^{D}\right)
$$

where $\Psi_{s}\left(x_{s}^{D}\right)=\sum_{n=1}^{N} \operatorname{Pr}(n) \Psi_{s}^{n}\left(x_{s}^{D}\right)$ and $\Omega_{i j}\left(y_{i j}^{D}\right)=\sum_{n=1}^{N} \operatorname{Pr}(n) \Omega_{i j}^{n}\left(y_{i j}^{D}\right)$.

Note that the two-stage stochastic program (1) - (13) is a nonlinear mixed integer stochastic program. To better understand the structure of the two-stage nonlinear mixed integer stochastic program, we rewrite the program as follows. Let $x^{D}=\left(x_{s}^{D}: s \in S\right)$ and $y^{D}=\left(y_{i j}^{D}:(i, j) \in \Pi\right)$. For the given first-stage decision variables $\left(x^{D}, y^{D}\right)$, the second-stage optimization problem is to choose $\left(G_{g}^{n k}, P_{i j}^{n k}, U_{i}^{n k}\right)$ to minimize

$$
\sum_{n=1}^{N} \operatorname{Pr}(n) \sum_{k=1,2,3,4}\left(\sum_{i \in B} c^{B} U_{i}^{n k}+\sum_{g \in G} c_{g}^{G} G_{g}^{n k}\right)\left(t_{k}-t_{k-1}\right)
$$

subject to constraints (2) - (12). Note that the second-stage optimization problem is a linear program and the program can be solved by scenario when the first stage decisions are known. Let $\Phi\left(x^{D}, y^{D}\right)$ be the objective function value associated with Equation (14) subject to constraints (2)-(12) for the given first stage variables $\left(x^{D}, y^{D}\right)$. Then the first-stage of the twostage nonlinear mixed integer stochastic program is to choose the binary variables $\left.\left(x^{D}, y^{D}\right)\right)$ to minimize

$$
\Phi\left(x^{D}, y^{D}\right)+\sum_{s \in S} \Psi_{s}\left(x_{s}^{D}\right)+\sum_{(i, j) \in \Pi} \Omega_{i j}\left(y_{i j}^{D}\right)
$$

subject to (1). Notice that this is a knapsack problem. This motivates our solution procedure described in the next section.

\section{SOLUTION PROCEDURE}

As mentioned previously, the first-stage problem is a knapsack problem with a nonlinear objective function. The key idea of our heuristic is to construct a knapsack problem with 
linear objective function so that the solution of the knapsack problem is also a good solution to the first-stage problem. The heuristic consists of four steps. The first step is to run the direct current (dc) economic dispatch model assuming all components are available. The second step is to run the dc economic dispatch assuming that a single component $\in \operatorname{set}(R)$ (set of all components that can be reinforced) is not functional. The third step is done for each component that can be reinforced. That step involves computing the relative benefit from reinforcing each component. The fourth step identifies the subset of reinforcement strategies that maximizes the benefit (as approximated using the weights developed in step 2) subject to the budget constraint given in equation (1). To simplify the notations, let us consider the following parametric de load flow dispatch problem where we determine $\left(\theta_{i}, G_{g}, P_{i j}, U_{i}\right)$ that minimizes

$$
\sum_{i \in B} c^{B} U_{i}+\sum_{g \in G} c_{g}^{G} G_{g}
$$

subject to

$$
\begin{gathered}
\left(\theta_{i}-\theta_{j}\right)\left(1-\tau_{i j}\right)\left(1-\varsigma_{s_{i}}\right)\left(1-\varsigma_{s_{j}}\right)=m_{i j} P_{i j}, \forall(i, j) \\
\sum_{g \in I(i)} G_{g}-\sum_{(i, j) \in \delta^{+}(i)} P_{i j}+\sum_{(i, j) \in \delta^{-}(i)} P_{i j}=D_{i}-U_{i}, \forall i \\
\left|P_{i j}\right| \leq P_{i j}^{m}\left(1-\tau_{i j}\right), \forall(i, j) \\
0 \leq U_{i} \leq D_{i}, \forall i \\
0 \leq G_{g} \leq G_{g}^{m}, \forall g
\end{gathered}
$$

where $\tau_{i j}$ and $\varsigma_{s_{i}}$ are input parameters for all $(i, j)$. The solution procedure is then as follows.

Step 1: Run the de flow economic dispatch problem (16) - (21) with $\tau_{i j}=\varsigma_{s_{i}}=\varsigma_{s_{j}}=0$ for all $(i, j)$ to determine $\left(\theta_{i}, G_{g}, P_{i j}, U_{i}\right)$. Note that the load shed at each bus $U_{i}=0$ since we assume that all components in the network are functional. Let $\bar{\lambda}$ be the optimal objective value.

Step 2: Let the $\operatorname{set}(R)$ be comprised of the collection of components $r$ for which there is at least one consequence scenario under which the component is not operational but with mitigation it becomes operational, for at least one time period. Run the de flow economic dispatch problem (16) - (21) for each component $r \in \operatorname{set}(R)$ assuming that component $r$ is not

\section{Romero - 9}


functional. To do this, the parameters in the program are set as follows. If $r=(i, j)$, we let $\tau_{i j}=1$ for $(i, j)=r$ and let $\tau_{i j}=0$ for $(i, j) \neq r$. We also let $\varsigma_{s_{i}}=\varsigma_{s_{j}}=0$ for all $(i, j)$. If $r=s_{i}$, we let $\varsigma_{s_{i}}=1$ for $s_{i}=r$ and let $\varsigma_{s_{i}}=0$ for $s_{i} \neq r$. We also let $\tau_{i j}=0$ for $(i, j)$. We determine the solution $\left(\theta_{i}, G_{g}, P_{i j}, U_{i}\right)$ and let $\lambda^{r}$ be the optimal objective value.

Step 3: Estimate the benefit of reinforcement for all components for which it is a consideration. Let $r \in \operatorname{set}(R)$ be the members of that set.

If $r=(i, j)$, let

$$
\beta_{i j}^{D}=\sum_{n=1}^{N} \operatorname{Pr}(n) \sum_{k=1,2}\left(\lambda^{i j}-\bar{\lambda}\right)\left(\Lambda_{i j}^{n k}(0)-\Lambda_{i j}^{n k}(1)\right)\left(t_{k}-t_{k-1}\right)+\Omega_{i j}(0)-\Omega_{i j}(1)
$$

If $r=s$, let

$$
\begin{aligned}
\beta_{s}^{D}=\sum_{n=1}^{N} \operatorname{Pr}(n) & \left(\sum_{k=1,2,3}\left(\lambda^{s}-\bar{\lambda}\right)\left(\Delta_{s}^{n k}(0)-\Delta_{s}^{n k}(1)\right)\left(t_{k}-t_{k-1}\right)\right) \\
& +\sum_{n=1}^{N} \operatorname{Pr}(n)\left(\left(\lambda^{s}-\bar{\lambda}\right) \bar{S}(s)\left(\Delta_{s}^{n 4}(0)-\Delta_{s}^{n 4}(1)\right)\left(t_{4}-t_{3}\right)\right)+\Psi_{s}(0)-\Psi_{s}(1)
\end{aligned}
$$

It is important to notice that in the fourth time period some large transformers may not be operational in some substations. The substation may still be used, but its performance has not been fully restored. To reflect this we define $\bar{S}(s)$ be the serviceability of a substation. The definition of this quantity is the fraction of lines and transformers that are functional within the substation to the total number of lines and transformers (both functional and nonfunctional) within the substation. Notice that the values $\lambda^{i j}$ and $\lambda^{s}$ are the result of step 2 . Also, $\Lambda_{i j}^{n k}(0)\left(\Delta_{s}^{n k}(0)\right)$ are binary input data that reflect whether a line (substation) is operational during period $k$ under scenario $n$ if no mitigation is performed. Similarly, $\Lambda_{i j}^{n k}(1)\left(\Delta_{s}^{n k}(1)\right)$ are binary input data that reflect whether a line (substation) is operational during period $k$ under scenario $n$ if mitigation is performed. Finally, $\Omega_{i j}(0)-\Omega_{i j}(1)$ reflects the repair costs savings if mitigation is performed on a line. Similarly, $\Psi_{s}(0)-\Psi_{s}(1)$ is the same quantity, except it is associated with substation repair cost savings.

Step 4: Determine the reinforcement strategy for the network. Run the integer problem to determine the reinforcement strategy $(x, y)$ that maximize

\section{Romero - 10}




$$
\sum_{s \in S} \beta_{s}^{D} x_{s}^{D}+\sum_{(i, j) \in \Pi} \beta_{i j}^{D} y_{i j}^{D}
$$

subject to

$$
\begin{gathered}
\sum_{s \in S} b_{s}^{D} x_{s}^{D}+\sum_{(i, j) \in \Pi} f_{i j}^{D} y_{i j}^{D} \leq M^{D} \\
x_{s}^{D}=0, \forall s \notin \operatorname{set}(R), y_{i j}^{D}=0, \forall(i, j) \notin \operatorname{set}(R)
\end{gathered}
$$

\section{CASE STUDY}

To illustrate the model and solution procedure described above, we focus on questions of seismic mitigation of the EI under limited budget. The representation of the EI is a 2003 Summer Peak ECAR (East Central Area Reliability Coordinating Agreement) case. The representation of the EI was developed in 1998 by the Multi-Area Modeling Working Group which is part of the Eastern Interconnection Reliability Assessment Group (https://rfirst.org/reliability/easterninterconnectionreliabilityassessmentgroup/Pages/default.as px). It is a representation of the system as of 1998 with demands reflective of a prediction of the summer of 2003. This case includes direct representation of every region in the EI. Detailed representation is only provided for voltages greater than $100 \mathrm{kV}$. It includes information for 23,416 transmission lines and 14,957 buses. These buses are grouped in 2,765 substations with two or more buses and 6,448 single buses. Load shed, generation output, repair, and mitigation costs were estimated in 2002 U.S. dollars.

We only consider the seismic risk from the NMSZ. In order to model the hazard, the first step is to develop a suite of earthquake scenarios (location and magnitude) that replicate important measures of the seismic hazard. For electric power systems, the key measure is the annual exceedance curves for PGA where seismically sensitive components are located. We located 81 control points in the NMSZ area and obtained the PGA exceedance curves for each point from the USGS Seismic Hazard Maps (USGS 2008b).

For our modeling, the hazard is represented by a set of earthquake scenarios (and their hazard-adjusted probabilities of occurrence) selected using the mathematical optimization method developed by Vaziri et al. (2012). The method which select the events and their hazard-adjusted occurrence probabilities so as to minimize the error between the annual exceedance probability of PGA curves implied by the selected scenarios, and the "true" PGA 
curves, which for this analysis are given in the USGS Seismic Hazard Maps (USGS 2008b). A key input to that optimization is the identification of the candidate set of earthquake events. We used two sources to create the candidate set: the earthquake catalog from the USGS website (USGS 2008) and a synthetic data set created by the USGS. This earthquake catalog includes 433 earthquakes that occurred within the NMSZ. The magnitudes were converted from $\mathrm{m}_{\mathrm{blg}}$ to $\mathrm{M}_{\mathrm{W}}$ using Atkinson and Boore (1995) and Johnston (1996) equally weighted as given in Petersen et al (2008). The mean PGA for each control point was estimated using Toro et al (1997), Frankel et al (1996), Campbell (2003), Atkinson and Boore (2006), Tavakoli and Pezeshk (2005), and Silva et al (2002) assuming soil type BC (shear-wave velocity, $760 \mathrm{~m} / \mathrm{s}$ ), the relative weights given in Petersen et al (2008). In addition to the 433 earthquakes identified in the Central-East Unites States earthquake scenarios catalog, we use 20 synthetic events on 5 synthetic faults created by USGS to refine the representation of the hazard in New Madrid. The 20 scenarios correspond to each of 4 possible magnitudes (7.3, 7.5, 7.7 and 8) for ruptures in the 5 different branches described in Petersen et al (2008). USGS (2008c) provides computer code that can be compiled and run to generate each of these deterministic scenarios in New Madrid.

Table 1 presents the 8 earthquake scenarios selected (from the 433 earthquake events in the historic catalogue and the 20 synthetic events developed by USGS) with their hazardadjusted occurrence probability. Figure 1 illustrates the annual exceedance curve from USGS and the estimated exceedance curve obtained from the selected scenarios (and their probabilities of occurrence) based on the formulation given in Vaziri et al. (2012) for a single control point located near the border between Tennessee and Arkansas at the Mississippi River near Osceola, Arkansas. Notice that there is very little difference in the estimated exceedance curves based on the 8 scenarios (and their adjusted probabilities of occurrence) and the exceedance information provided by USGS.

Table 1. Selected earthquake events and their probabilities of occurrence.

\begin{tabular}{|c|c|c|c|c|c|c|c|c|}
\hline \multicolumn{3}{|c|}{ "Location } & \multirow{2}{*}{$\begin{array}{l}\text { Depth } \\
{[\mathrm{km}]}\end{array}$} & \multicolumn{2}{|c|}{ Magnitude } & \multirow[b]{2}{*}{ Date } & \multirow[b]{2}{*}{ Source } & \multirow{2}{*}{$\begin{array}{l}\text { Hazard- } \\
\text { adjusted } \\
\text { occurrence } \\
\text { probability }\end{array}$} \\
\hline Lat. & Long. & $\begin{array}{c}\text { Fault } \\
\text { Info. }\end{array}$ & & $\mathbf{m}_{\text {blg }}$ & $\mathbf{M}_{\mathbf{W}}$ & & & \\
\hline 36.7 & -90.3 & & 0.0 & 4.3 & & $2 / 2 / 1954$ & NCEER & 0.0500 \\
\hline 38.2 & -89.8 & & 11.0 & 4.3 & & 4/9/1955 & NCEER & 0.0500 \\
\hline 37.9 & -88.4 & & 21.0 & 5.5 & & $11 / 9 / 1968$ & NCEER & 0.0078 \\
\hline 38.7 & -88.0 & & 10.0 & 5.2 & & $6 / 10 / 1987$ & USHIS|5.20mn & 0.0069 \\
\hline
\end{tabular}




\begin{tabular}{l|l|l|l|l|l|l|l|l}
36.8 & -89.2 & & 5.0 & 4.5 & & $9 / 29 / 1987$ & USHIS|4.50mn & 0.0500 \\
35.8 & -90.2 & & 9.0 & 4.2 & & $5 / 1 / 2005$ & PDE|4.20mw & 0.0500 \\
& & Mid-East & & & 7.7 & & USGS faults & 0.0018 \\
& & & & 8.0 & & USGS faults & 0.0010 \\
\hline \hline
\end{tabular}

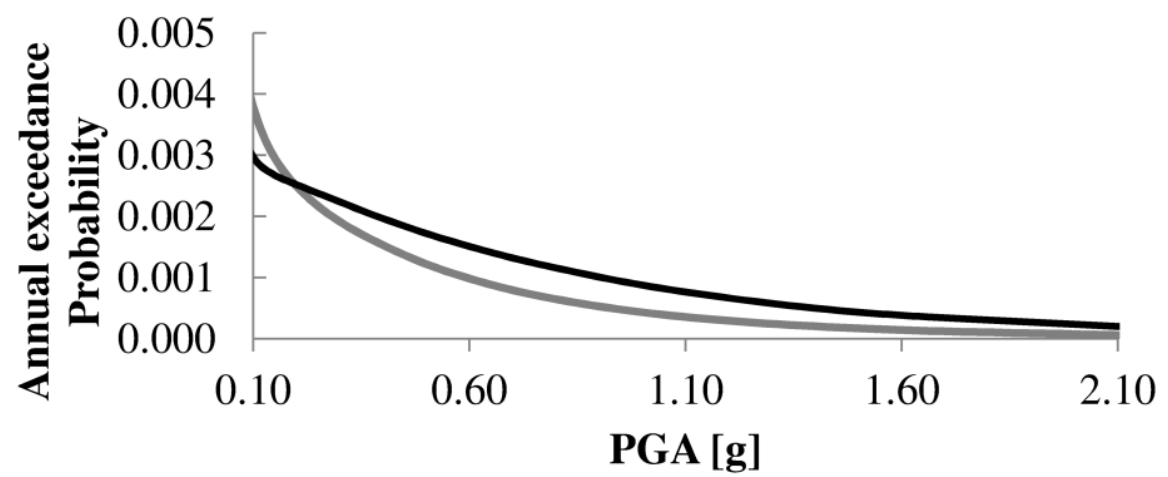

-USGS -Estimated from subset of scenarios

Figure 1. Annual exceedance curves for location P84, control point located at the border between Tennessee and Arkansas at the Mississippi River near Osceola, Arkansas.

Figure 2 gives a histogram of the difference between the PGA for each of the return periods based on the USGS data at each location and the probability based on the earthquakes selected for that same value of PGA. It is useful to notice that the errors are quite small and the distribution of errors is symmetric indicating that the approximation of the exceedance curves is not biased.

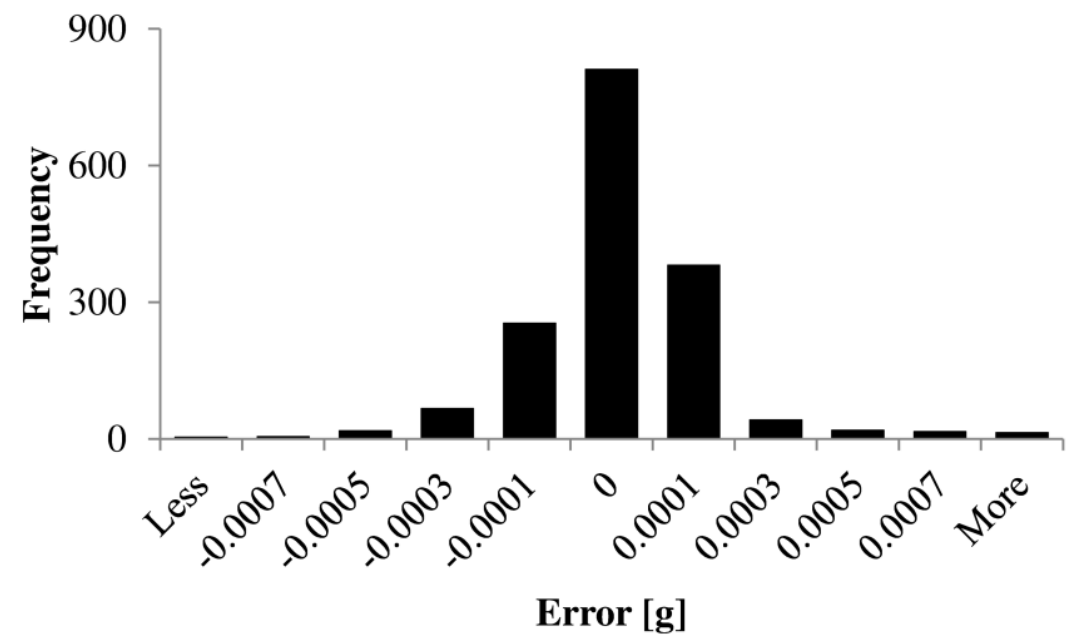

Figure 2. Histogram of PGA optimization error for every combination of location and return period. 
Under each scenario the probability that each component sustains specific levels of damage is then computed using a regional loss estimation methodology, in this case HAZUS (FEMA 2003). HAZUS categorizes damage to substations and transmission lines into five classes: no damage, slight, moderate, extensive and complete. HAZUS defines the probability that a component sustains each of five damage stages after an earthquake of a certain intensity, and considering if the component was seismically retrofitted or not. As mentioned before we only modeled moderate, extensive and complete damage for substations and extensive and complete for transmission lines. For the analysis in this paper, we assume that none of the substation components have been anchored, and that transmission towers have not been seismically reinforced. This assumption is based on data availability not intrinsic limitations in the modeling process.

Since the performance of a network is governed by the distribution of damage over all components, each earthquake scenario must be translated into a set of consequence scenarios. In each consequence scenario, the level of damage of each component is specified. More generally, we develop these consequence scenarios specifying the condition for each component with and without seismic mitigation, which for transmission lines is seismic retrofit and for substation components, anchorage of subcomponents. Each consequence scenario is identified by a revised-adjusted occurrence probability, which combines the probability of the occurrence of the earthquake scenario and the probability of the damage level for each component from that earthquake scenario.

We use the optimization method introduced by Brown et al. (2011) to develop consequence scenarios and their probability of occurrence. Each consequence scenario has a realized damage state for each component of the infrastructure and an associated probability. The objective of the optimization is to select a defined number of consequence scenarios and occurrence probabilities so that when all the consequence scenarios are combined the estimated vulnerabilities of each component match the "true" (input) vulnerability as closely as possible. In this case, the "true" vulnerability is defined by HAZUS fragility curves (FEMA 2003). For the purposes of this research, the Brown et al. (2011) optimization model is expanded to include the performance of the components prior to reinforcement and after reinforcement.

The six events with $m_{\text {blg }}$ less than 6 (See Table 1) result in effectively no physical damage to the electric power grid based on the fragility curves given in HAZUS and the probability 
distribution for PGA for each event at each location (FEMA 2003). The earthquake scenario on the Mid-East fault of magnitude $7.7 \mathrm{M}_{\mathrm{W}}$, and the earthquake scenario on the West fault of magnitude $8.0 \mathrm{M}_{\mathrm{W}}$ do result in considerable damage. For each of these events, we generate 6 consequence scenarios. Notice that the sum of the revised-adjusted occurrence probabilities of each of these two sets of scenarios in Table 2 equals the corresponding hazard-adjusted occurrence probably for the event scenario given in Table 1. The average error across both earthquake scenarios for the probability that each component is in each of the damage states is less than 7\%. Table 2 presents the 12 consequence scenarios and the number of transmission lines and substations that fall into each of the possible damage states. The third column presents the probability that we assigned to each scenario to match the ground motion hazard probability and the components' vulnerability. Figure 3 shows the spatial distribution of PGA for the earthquake scenario in the West branch with a magnitude of $8.0 \mathrm{M}_{\mathrm{W}}$ and the consequence scenario 6 (See ID column in Table 2). It is useful to notice that there is substantial damage across the New Madrid area including in Memphis, TN, Evansville, IN and St. Louis, IL. Figure 4 gives the estimated load shed for that same event. Again, the impacts across the region are very significant, especially around Memphis, which is the nearest city to the earthquake rupture.

Table 2. Consequence scenarios use to represent the NMSZ hazard on the EI.

\begin{tabular}{|c|c|c|c|c|c|c|c|c|c|c|c|c|}
\hline \multirow{3}{*}{ Source } & \multirow{3}{*}{ ID } & \multirow{3}{*}{$\begin{array}{c}\text { revised- } \\
\text { adjusted } \\
\text { occurrence } \\
\text { probability }\end{array}$} & \multicolumn{5}{|c|}{ Basic design } & \multicolumn{5}{|c|}{ Seismically reinforced components } \\
\hline & & & \multicolumn{2}{|c|}{$\begin{array}{l}\text { Transmission } \\
\text { Lines damage }\end{array}$} & \multicolumn{3}{|c|}{$\begin{array}{l}\text { Substations } \\
\text { damage }\end{array}$} & \multicolumn{2}{|c|}{$\begin{array}{l}\text { Transmission } \\
\text { Lines damage }\end{array}$} & \multicolumn{3}{|c|}{ Substations damage } \\
\hline & & & Ext. & Com. & Mod. & Ext. & Com. & Ext. & Com. & Mod. & Ext. & Com. \\
\hline \multirow{6}{*}{$\begin{array}{c}\text { West } \\
\text { branch, } \\
8.0 \mathrm{M}_{\mathrm{W}}\end{array}$} & 1 & 0.000160 & 5 & 24 & 18 & 8 & 15 & 2 & 24 & 15 & 3 & 7 \\
\hline & 2 & 0.000200 & 5 & 25 & 23 & 19 & 8 & 6 & 20 & 13 & 15 & 7 \\
\hline & 3 & 0.000080 & 15 & 24 & 68 & 24 & 10 & 25 & 5 & 66 & 26 & 9 \\
\hline & 4 & 0.000180 & 4 & 25 & 13 & 14 & 10 & 5 & 20 & 7 & 12 & 7 \\
\hline & 5 & 0.000240 & 6 & 24 & 12 & 16 & 9 & 4 & 22 & 8 & 20 & 6 \\
\hline & 6 & 0.000140 & 6 & 22 & 26 & 22 & 12 & 4 & 21 & 26 & 12 & 6 \\
\hline \multirow{6}{*}{$\begin{array}{c}\text { Mid-East } \\
\text { branch, } \\
7.7 \mathrm{M}_{\mathrm{W}}\end{array}$} & 7 & 0.000126 & 27 & 11 & 35 & 16 & 14 & 21 & 9 & 29 & 13 & 14 \\
\hline & 8 & 0.000396 & 28 & 14 & 4 & 13 & 11 & 16 & 6 & 3 & 13 & 9 \\
\hline & 9 & 0.000288 & 30 & 13 & 17 & 12 & 12 & 19 & 8 & 9 & 8 & 11 \\
\hline & 10 & 0.000342 & 28 & 13 & 6 & 7 & 19 & 24 & 8 & 9 & 10 & 11 \\
\hline & 11 & 0.000432 & 24 & 14 & 5 & 13 & 13 & 18 & 8 & 3 & 19 & 6 \\
\hline & 12 & 0.000216 & 28 & 26 & 25 & 10 & 13 & 15 & 9 & 13 & 16 & 5 \\
\hline
\end{tabular}






Figure 3. Distribution of PGA and damage for an $8.0 \mathrm{M}_{\mathrm{W}}$ earthquake on the West Branch under consequence scenario 6 . 


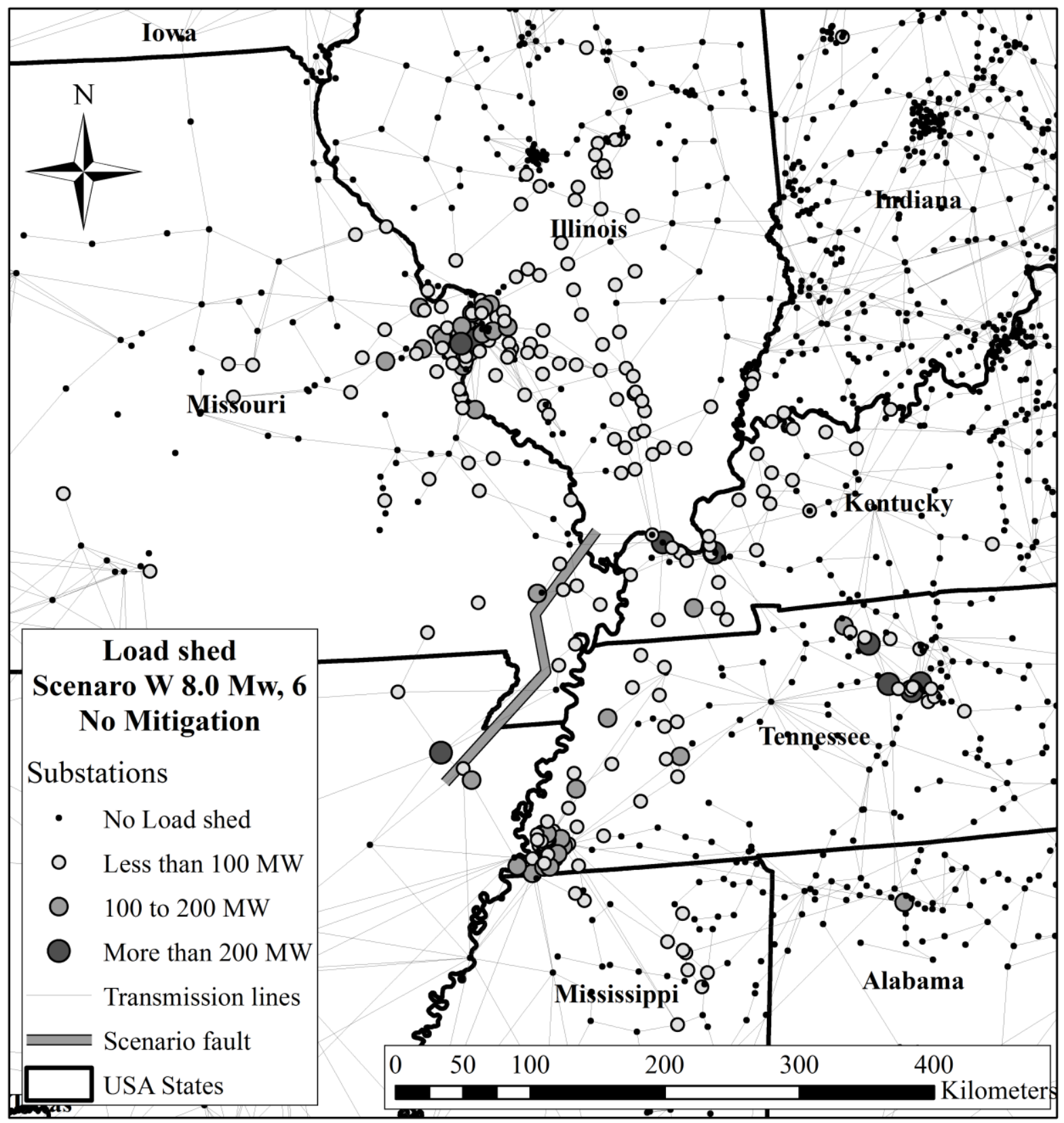

Figure 4. Estimated load shed for an $8.0 \mathrm{M}_{\mathrm{W}}$ earthquake on the West Branch under consequence scenario 6.

\section{RESULTS}

The proposed solution procedure was tested using a simplified version of the model which considers only the first time period and two of the twelve earthquake scenarios. We solved the nonlinear integer programming problem (NLIP) for 3 different mitigation budgets: US\$100 million, US\$20 million, and US\$10 million using two methods: the proposed solution procedure coded in C++ with IBM ILOG Optimization Studio CPLEX 12.2 (IBM Corporation 2010) serving as an LP solver, and the full NLIP in LINGO 13 (Lindo System 
Inc. 2011) -which has a non-linear integer solver- in a Dell Precision T5500, Intel® Xeon® X5650 with 2 processors of $2.66 \mathrm{GHz}$., and $6.00 \mathrm{~GB}$ of total RAM memory. LINGO found a solution with $0.5 \%$ lower total seismic risk costs solution for a mitigation budget of US\$100 million; for the other two problem instances the solution procedure described above found solutions that resulted in $20 \%$ lower repair and load shed costs. LINGO took over 8 hours to solve and the solution procedure described above took 8 minutes. Given the computational burden, LINGO cannot be used to address the full problem formulation for the EI.

We used the heuristic to find a seismic risk mitigation strategy for the full problem formulation for the EI using the 12 consequence scenarios identified to represent the NMSZ hazard. EI performance after an event was modeled using the 4 time periods as defined by the repair times for the various components described above. We assume about 50 lines and 110 substations are viable candidates for reinforcement. The total running time varies depending on the budget; the average computation time is 1 hour using the machine described above.

Figure 5 shows the annual exceedance probability of load shed costs during repair for 5 different investment scenarios: no-mitigation, US\$ 5 million, US\$ 10 million, US\$ 20 million, and US\$ 50 million, respectively. For a budget of US\$ 5 million, the model suggests no seismic reinforcement for lines, and that about $63 \%$ of substations should be anchored. For a budget of US\$ 10 million, $6 \%$ of transmission lines and $87 \%$ of substations are selected for reinforcement. For US\$ 20 million, about 12\% of transmission lines and $98 \%$ of substation are reinforced. Finally, for US\$ 50 million, 36\% of transmission lines and 100\% of substations should be reinforced. It is useful to notice that the optimization favors investments in substations over transmission lines. This is because substations take considerably longer to repair than lines. 


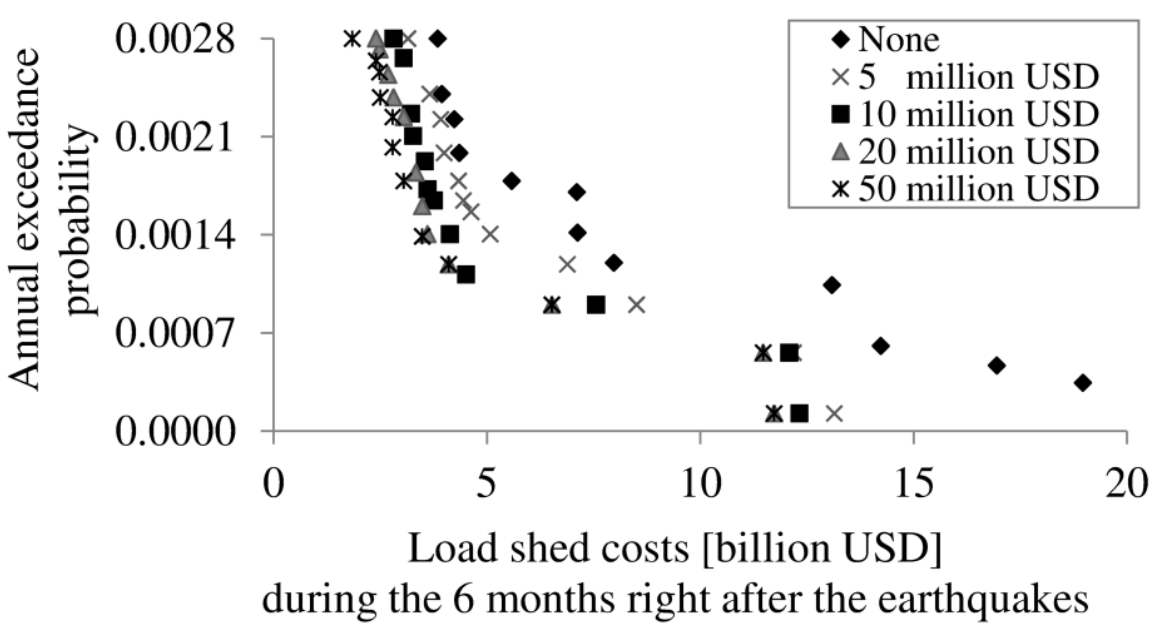

Figure 5. Annual exceedance probability for load shed costs during repair.

Figure 6 shows the repair costs annual exceedance probabilities. Investing US\$10 million can reduce the load shed costs by almost $40 \%$, and the repair costs by almost $20 \%$. In expectation, this reduction translates to a saving of about US\$ 12 million annually stemming from an upfront investment of US\$ 10 million. Figure 7 gives the load shed based on the recommended mitigation when the budget is US\$10 million. It is useful to notice that, in contrast to Figure 4, there is substantially less load shed, particularly around the St Louis Area. The event is so severe in the Memphis area that mitigation has little effect. However in the areas that are more removed from the event, the insult is significantly less severe so the mitigation has a more pronounced effect. For example the load shed is reduced by about $45 \%$ in the area that is between $100 \mathrm{~km}$ and $200 \mathrm{~km}$ from the fault rupture.

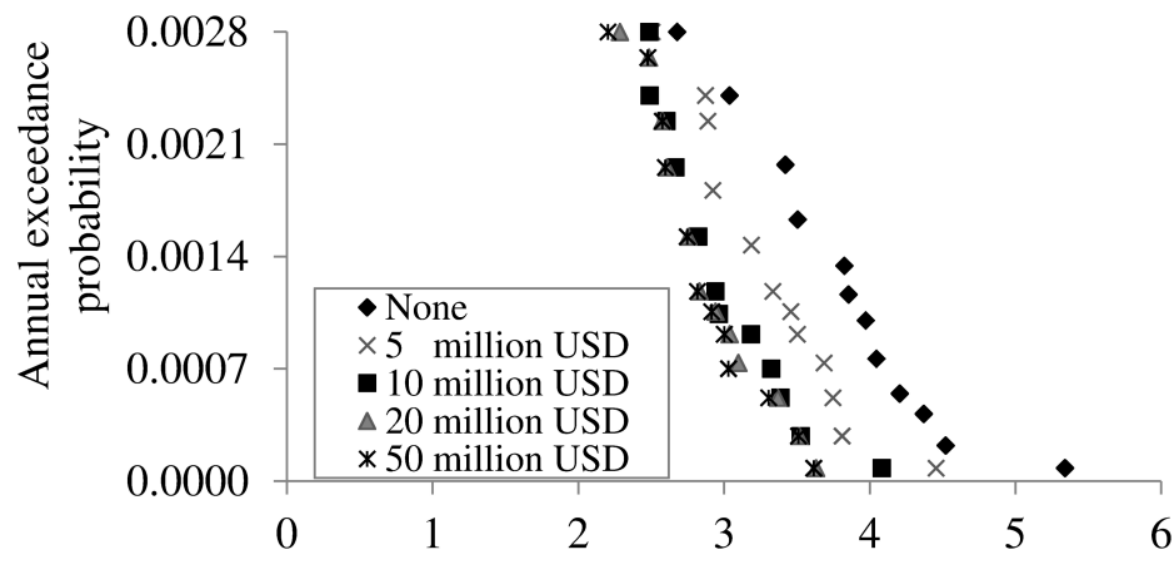

Repair costs [billion USD] after the earthquakes

Figure 6. Repair costs annual exceedance probability.

$$
\text { Romero - } 19
$$




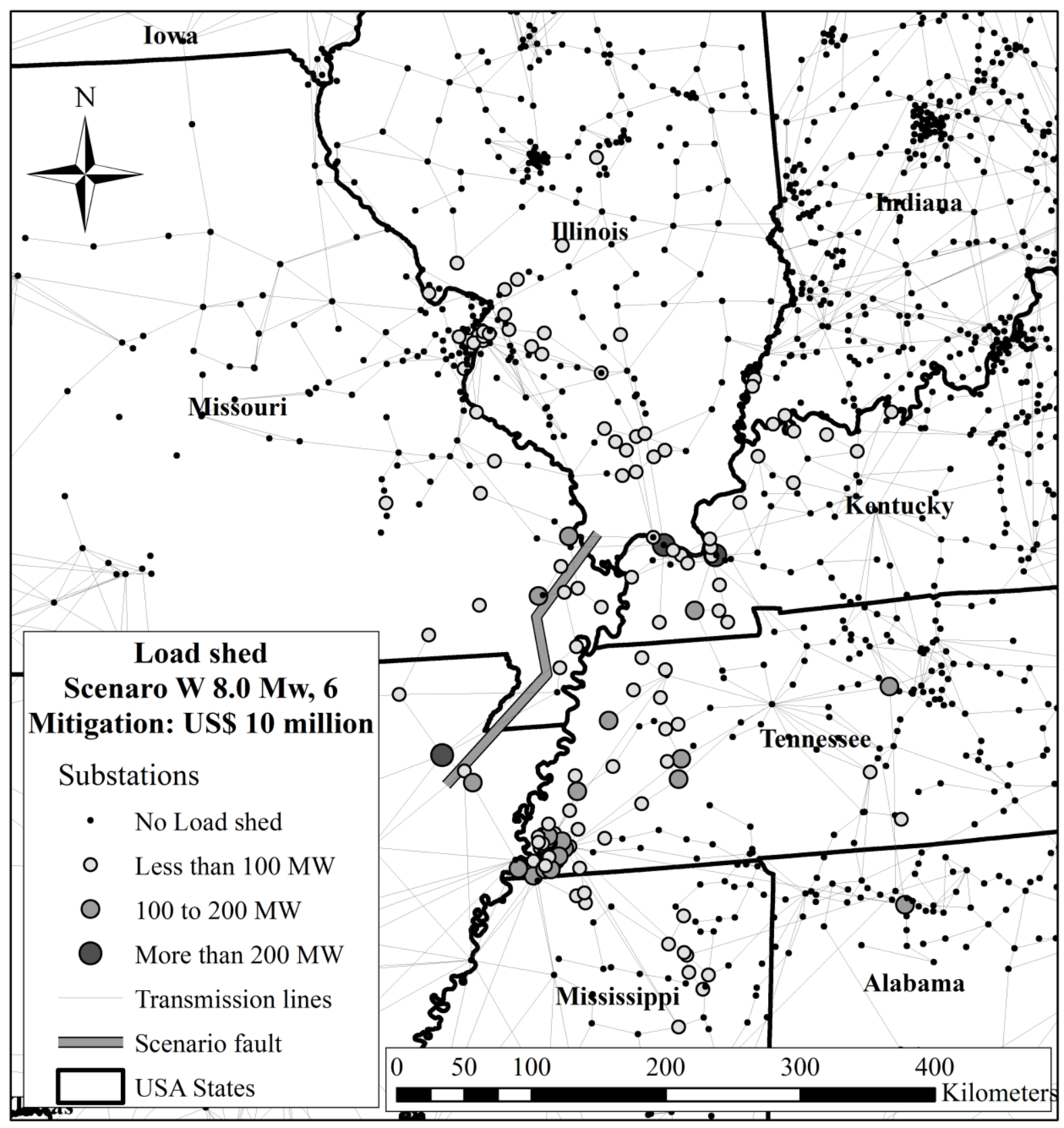

Figure 7. Load shed with seismic reinforcement the seismic reinforcement recommended under a budget constraint of US \$10 million under consequence scenario 6.

\section{CONCLUSIONS}

This paper has developed a method, using two-stage stochastic programming, to identify an approximately optimal seismic mitigation strategy for electric power system components that can be used to address large scale problem instances. It makes use of methods already developed to create a range of seismic events (location and magnitude) and their hazardconsistent probabilities to characterize the hazard. For each of these events, consequence 
scenarios and their probability of occurrence are developed to understand the distribution of damage under that event and the level of degradation in system performance. These two steps provide a robust mechanism to understand the impacts of earthquakes on the system and how that impact might be modified through specific investments. The remainder of the paper developed an optimization method to understand how to optimize the selection of mitigation strategies under a budget constraint.

More specifically, a two-stage stochastic program was developed. The first stage identifies the mitigation strategies to perform, whereas the second stage is effectively an economic dispatch model of the electric power system to compute the repair costs and the load shed under each consequence scenario, based on the investments made. In the case study developed in this paper, the regional loss estimation methodology, HAZUS (FEMA 2003), was used to compute the probability that each component fell into each of a set of mutually exclusive and exhaustive damage states for each consequence scenario. The method was applied to the EI, which has almost 15,000 nodes and 23,000 links, and the seismic hazard considered stems from the NMSZ.

There are a range of opportunities for further research. First, the key mitigation strategy considered in this paper is the anchoring of components. There is also the opportunity to add capacity to the existing network to increase earthquake resilience. How to add this additional capacity then becomes the subject of an optimization. Also, we do not consider the question of the optimization of spare transformers including which ones to hold in inventory pre-event and how they should be deployed post-event. One of the key benefits of addressing seismic hazards via capacity augmentation is that the added capacity is valuable under a range of circumstances beyond addressing the consequences of earthquakes including periods of peak demand (heat waves, etc.), hurricanes and ice storms. Moreover, under normal operation, the added capacity can enable cheaper, cleaner or more sustainable generation or increased reliability.

Second, the assessment of the impacts of each of the consequence scenarios based on the mitigation performed did not include the potential for further cascading beyond the initial damage. While the cascading usually involves outages that do not damage components, it can lead to a more widespread load shed (blackouts) that can delay the recovery from the earthquake. Modeling cascades in electric power systems stemming from earthquakes is complicated, and approximate methods are beginning to be developed (Kim and Dobson

$$
\text { Romero - } 21
$$


2010). Cascades occur when the system is fragile and in a damaged state. Hence, consideration of the potential for cascades when developing mitigation strategies for electric power systems may turn out to be important.

Third, the input data does not capture damage from local site conditions, or the specific vulnerability of each component. Detailed soil information for the study, structural design of the components located in critical areas, and specific structural retrofitting options for each component would be valuable contributions to a dataset for a model of this nature.

Finally, the electric power system is critical to the operation of many other infrastructures. Conversely, the electric power system is dependent on other infrastructures. Understanding the impact of earthquakes on interdependent infrastructures (including electric power networks) is very important. In the context of mitigation, this avenue for additional research becomes even more compelling.

\section{ACKNOWLEDGEMENTS}

Ian Dobson gratefully acknowledges funding in part from DOE grant DE-SC0002283

\section{REFERENCES}

Atkinson, G. M., and Boore, D. M., 1995. Ground-Motion relations for eastern North America. Bulletin of the Seismological Society of America 85, 17 - 30.

Atkinson, G. M., and Boore, D. M., 2006. Earthquake ground motion prediction equations for eastern North America, Bulletin of the Seismological Society of America 96, 2181 - 2205.

Balducci, P. J., Schienbein, L. A., Nguyen, T.B., Brown, D. R. and Fathelrahman, E. M. 2006. An examination of the costs and critical characteristics of electric utility distribution system capacity enhancement projects in Proc. Transmission and Distribution Conference and Exhibition, pp. 78 $-86$.

Billington, R, Kumar, S., Chowdhury, N., Chu, K., and Debnath, K. 1989. A reliability test system for educational purposes - basic data. IEEE Transactions on Power Systems 4, 1238 - 1244.

Brown, N. J. K., Gearhart, J. L., Jones, D. A., Nozick, L. K., Xu, N., and Romero, N., 2011. Optimizing the selection of scenarios for loss estimation in transportation networks in Proceedings of the 2011 WSC: Simulation for a Sustainable World. Phoenix, Arizona.

Campbell, K. W., 2003. Prediction of strong ground motion using the hybrid empirical method and its use in the development of ground-motion (attenuation) relations in eastern North America. Bulletin of the Seismological Society of America 93, 1012 - 1033.

Dong, X., Shinozuka, M., and Change, S., 2004. Power network systems in Proceedings of the 13th World Conference on Earthquake Engineering, Vancouver, Canada.

Eidinger, J., 2009. Wenchuan Earthquake Impact to Power Systems in Proceedings of the 2008 TCLEE: Lifeline Earthquake Engineering in a Multihazard Environment, Oakland, California.

Federal Emergency Management Agency, Department of Homeland Security, 2003. HAZUS-MH Multi-hazard loss estimation methodology, earthquake model - Technical Manual. Washington, D.C.

\section{Romero - 22}


Frankel, A., Mueller, C., Bamhard, T., Perkins, D., Leyendecker, E., Dickman, N., Hanson, S., and Hopper, M., 1996. National Seismic Hazard Maps: Documentation U. S. Geological Survey, Report 96-532.

Gomberg, J., and Schweig, E., 2007. Understanding Earthquake Hazards in the Central United States, Earthquake Hazard in the Heart of the Homeland, U. S. Geological Survey, Fact Sheet 20063125.

IBM Corporation, 2010. ILOG Optimization Studio CPLEX 12.2. Armonk, NY.

Johnston, A. C., 1996. Seismic moment assessment of earthquakes in stable continental regions-I. Instrumental seismicity. Geophysical Journal International 124, 381 - 414.

Kim, J. and Dobson, I., 2010. Approximating a loading-dependent cascading failure model with a branching process, IEEE Transactions on Reliability 59, 691 - 699.

Lindo System Inc., 2011 Lingo 13.0, software. Chicago, IL.

Long, G., 2010 Reconectando a Chile después del terremoto, Revista Business Chile. < http://businesschile.cl/es/noticia/terremoto/reconectando-chile-despues-del-terremoto >

Noda, M., 2001. Disaster and restoration of electricity supply system by Hanshin-Awaji Earthquake in Proceeding of the Seminar on Earthquake Disaster Management of Energy Supply System. Chinese Taipei: Earthquake Response Cooperation Program for Energy Supply Systems.

Obermeier, S. F., Gohn, G. S., Weems, R. E., Gelinas, G. L., and Meyer, R., 1985. Geologic evidence for recurrent moderate to large earthquakes near Charleston, South Carolina, Science 227, 408 411.

Petersen, M. D., Frankel, A. D., Harmsen, S. C., Mueller, C. S., Haller, K. M., Wheeler, R. L., Wesson, R. L., Zeng, Y., Boyd, O. S., Perkins, D. M., Luco, N., Field, E. H., Wills, C. J., and Rukstales, K. S. 2008. Documentation for the 2008 update of the United States national seismic hazard maps. U. S. Geological Survey, Report 2008-1128.

Shinozuka, M., Feng, M., Dong, X., Chang, S., Cheng, T., Jin, X. and Saadeghvaziri, M. A., 2003. Advances in seismic performance evaluation of power systems, Resilience of Electric Power Substation Equipment, MCEER, Buffalo, New York.

Shumuta, Y. 2007. Practical seismic upgrade strategy for substation equipment based on performances indices. Earthquake Engineering and Structural Dynamics 36, 209 - 226.

Shumuta, Y., 2011. Tohoku Chiho-Taiheiyo-Oki Earthquake - Damage of electric power facilities in Tohoku Electric Power Co., Inc. Central Research Institute of Electric Power Industry, Civil Engineering Lab.

Silva, W., Gregor, N., and Darragh, R., 2002. Development of regional hard rock attenuation relations for central and eastern North America, Pacific Engineering, El Cerrito, CA.

Somerville, P., Collins, N., and Abrahamson, N., Graves, R., and Saikia, C., 2001. Ground motion attenuation relations for the Central and Eastern United States, Final Report to the U.S. Geological Survey, Contract No. 99HQGR0098.

Tavakoli, B., and Pezeshk, S., 2005. Empirical-stochastic ground-motion prediction for eastern North America. Bulletin of the Seismological Society of America 95, 2283 - 2296.

Toro, G. R., Abrahamson, N. A., and Schneider, J. F., 1997. A model of strong ground motions from earthquakes in central and eastern North America: Best estimates and uncertainties. Seismological Society of America 68, 41 - 57.

Tuttle, M. P., Schweig, E. S., Sims, J. D., Lafferty, R. H., Wolf, L.W., and Hayes, M. L., 2002. The earthquake potential of the New Madrid seismic zone, Bulletin of the Seismological Society of America 92, 2080 - 2089.

U. S. Department of Energy, 2009. Electric Power Annual, Energy Information Administration, Washington, D.C.

U. S. Geological Survey, 2008a. CEUS Catalog \#3: emb.cc, National Seismic Hazard Maps Catalogs,. <http://earthquake.usgs.gov/hazards/products/conterminous/2008/catalogs/>.

U. S. Geological Survey. 2008b. National Seismic Hazard Maps Gridded Data, Peak Ground Acceleration Hazard

Curve. <http://earthquake.usgs.gov/hazards/products/conterminous/2008/data/>

U. S. Geological Survey, 2008c. National Seismic Hazard Maps Software. 
http://earthquake.usgs.gov/hazards/products/conterminous/2008/software

Vanzi, I., 2000. Structural upgrading strategy for electric power networks under seismic action. Earthquake Engineering and Structural Dynamics 29. 1053 - 1073.

Vaziri, P., Davidson, R., and Nozick, L., 2012. Identification of optimized-based probabilistic earthquake scenarios for regional loss estimation. Journal of Earthquake Engineering 16, 296 315. 